\title{
Le Rhône à Lyon \\ Etude de l'entraînement des galets à l'aide de traceurs radioactifs
}

\author{
Essai pour l'extension de la formule de Meyer-Peter \\ au domaine du charriage partiel
}

\section{A study of pebble movements in the Rhone by means of radioactive tracers}

\author{
An attempt to extend the Meyer-Peter formula to the partial transport range
}

PAR

MM. RAMETTE ET HEUZEL

iNgÉnieurs aU taboratoire National D'hydraUliQUe, chatou

\begin{abstract}
Illustration des techniques de «marquage» des galets d̀ l'aide d'éléments de tantale radionctivés, d'immersion, puzis de détection par un film réalisé da l'occasion d'une expérience effectuée dans le Rhône pen en aval du confuent de la Saône.

Examen du cheminement des galets detectés après des crues du Rhốre par une série de campagnes. Détermination des forces tractrices de début d'entrainement en fonction des dimensions des galets qui varient entre 25 et $75 \mathrm{~mm}$. Critère de début de charriage total à partir duquel les formules de charriage de MeyerPeter sont applicables. Dans le domaine du charriage partiel, possibilité de donner un ordre de grandeur du débit solide par l'appllcation des formules de Meyer-Peter $\dot{a}$ différentes tranches granulométriques, connaissant les conditions de début d'entrainement des éléments constituant ces tranches.
\end{abstract}

Dans le cadre des problèmes hydrauliques posés par l'aménagement de la chute de PierreBénite, la Compagnie Nationale du Rhône a demandé au Laboratoire de Chatou d'étudier les transports solides par charriage sur les premiers kilomètres du Rhône en aval de son confluent avec la Saòne.

Des problèmes identiques s'étaient posés à l'occasion de l'élude des aménagements du tiers central du Rhône à Donzère-Mondragon, Montélimar, Baix, etc. Ils avaient été résolus d'une

\begin{abstract}
Deseription of techniques used in an experiment in the Rhone a short distance below its confluence with the Saone, whereby pebbles were marked with radio-active tantalum. placed in the river, and then followed by a photo-detection method.

Examination of recorded pebble travel data resulting from a series of measurements in the Rhone after a high water period. Determination of the forces required to set various pebble sizes in motion (between $25 \mathrm{~mm}$ and $75 \mathrm{~mm}$ ). Identification of an initial total transport criterion, as from which the Meyer-Peter formulae are applicable. Within the partial transport range, an approximate value can be determined for the solid flow rate by applying the Meyerpeter formulae to various grain size ranges, providing the conditions for the initial setting in motion of the materials in these ranges are known.
\end{abstract}

façon satisfaisante à partir des formules expérimentales de charriage de Meyer-Peter (1).

C'est donc à cette même formule que nous pensions recourir pour cette nouvelle étude.

Un des termes importants de la formule de Meyer-Peter est la force tractrice de début de

(1) Communication au $7^{\circ}$ Congrès de l'A.I.R.H. à Lisbonne en juillet 1957, par MM. GemaghulNg, Givocchro, Chabent : «Comparaison entre Ies évaluations théoriques et expérimentales du débit solide dans le tiers central du Rhône». 
charriage généralisé : $\tau_{0}$ qui entre dans la formule connue : $g=k\left(\tau-\tau_{0}\right)^{3 / 2}$. En général on adopte comme valeur de $\tau_{0}$ la force tractrice correspondant à un débit tel qu'un appareil, du genre hydrophone par exemple, détecte un déplacoment de fond des galets. Dire que ce déplacement affecte tous les galets est dans ces conditions difficile sinon impossible à déterminer; $\tau_{0}$ est donc connu assez approximativement; une telle approximation a en fait assez peu d'importance si les valeurs de $\tau$ sont très supérieures à $\tau_{i j}$. II en est hien ainsi dans le tiers central du Rhòne; dans la région lyonnaise par contre, les pentes du fleuve, et de ce fait les forces tractrices, sont beaucoup plus faibles; $\tau_{0}$ devait donc être déterminé avec plus de précision.

La méthode dite des traceurs permet de déterminer $\tau_{0}$; elle consiste à marquer des galets pour permettre d'en suivre les déplacements; si les dimensions des galets marqués couvrent l'étendue granulométrique des alluvions, la valeur de $\tau_{0}$ cherchée correspondra au débit minimal pour lequel tous les galets seront en mouvement.

\section{I. - Marquage des galets}

Les galets dont on veut suivre le déplacement sont «marqués» à l'aide d'un élément de tantale 182 radioactivé. Cet élément, qui se présente sous la forme d'un fil de $5 / 10^{\mathrm{es}} \mathrm{de} \mathrm{mm}$ de diametre et $1 \mathrm{~cm}$ de longueur environ, est introduit dans un trou borgne percé dans le galet; le trou est ensuite obturé par de la paraffine.

Le tantale radioactivé émet un rayonnement gamma; sa période est de 111 jours; l'expérience montre qu'un galet «marqué » par le tantale est reperable pendant plus de 6 mois à l'aide d'un détecteur classique : compteur Geiger, oscillomètre, etc.

\section{II. - Dimension et caractéristiques des galets ( marqués )}

Les galets marqués sont divisés en trois catégories, dont les caractéristiques sont les suivantes :

\begin{tabular}{|c|c|c|}
\hline Catégonies & $\begin{array}{l}\text { DMENSIONS } \\
\text { MOYENNES } \\
\text { SUIVANT } \\
\text { LES TROIS AXES } \\
\text { PRINCIPAUX } \\
\text { (mm) }\end{array}$ & $\begin{array}{c}\text { Nombre } \\
\text { DE } \\
\text { GALETS } \\
\text { MARQUÉs }\end{array}$ \\
\hline I - Gros galets... & $80 \quad 60 \quad 40$ & 30 \\
\hline II -- Galets moyens. & $55 \quad 40 \quad 30$ & 125 \\
\hline III - Petits galets... & $30 \quad 24 \quad 15$ & 250 \\
\hline
\end{tabular}

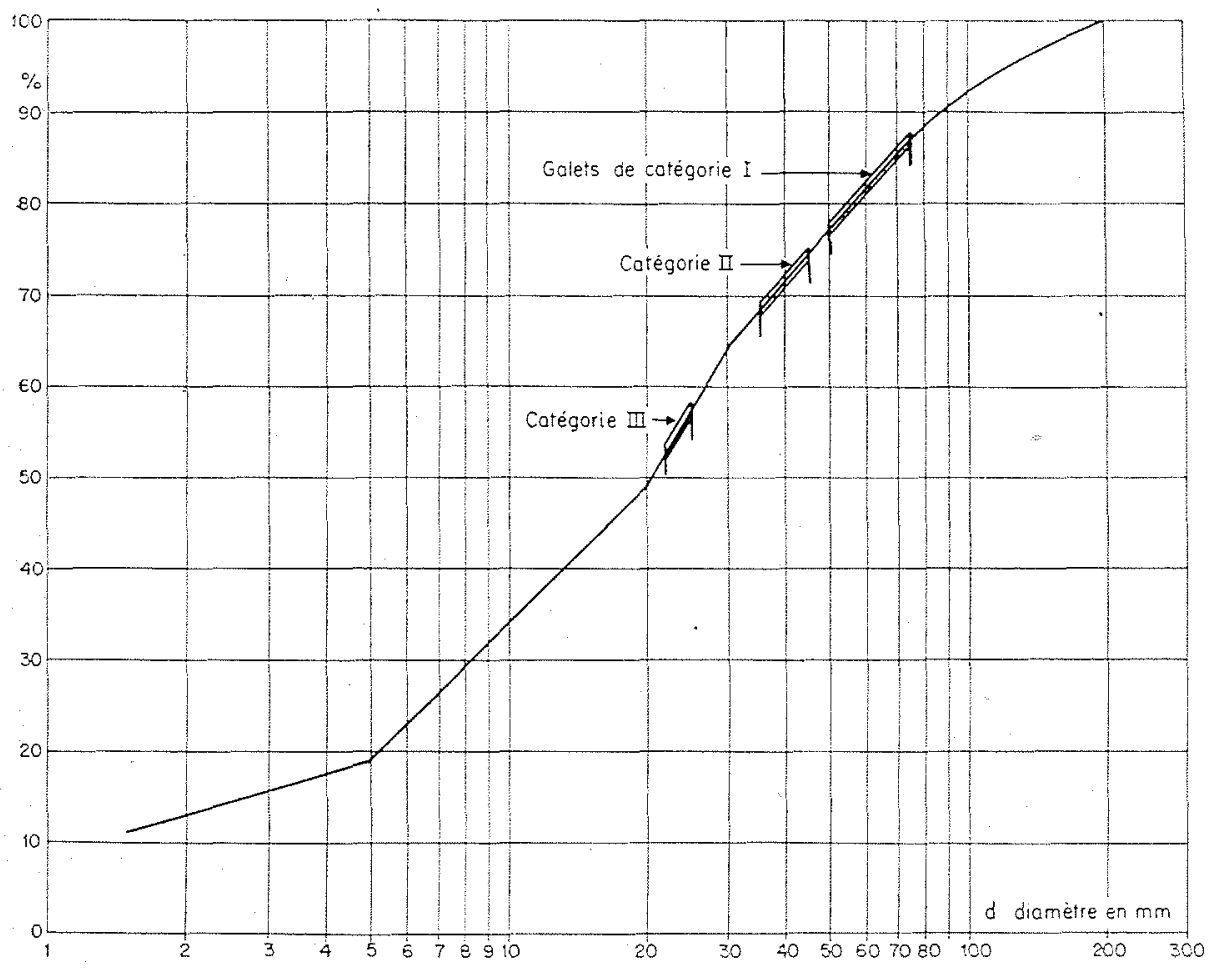

Fig. 1

Courbe granulométrique du Rhône

à Pierre-Bénite. 

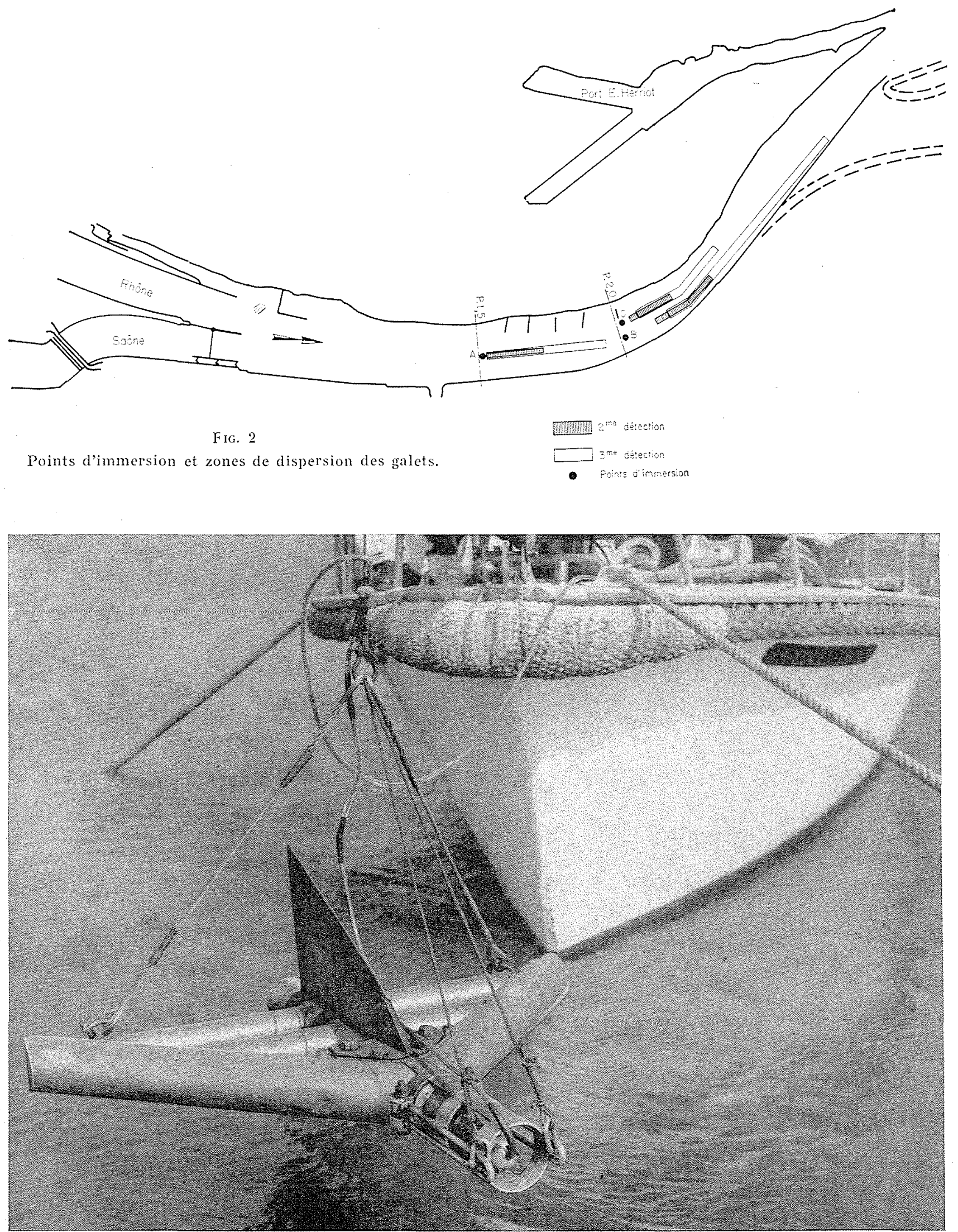

FIG. 3 
Les diamètres intermédiaires $60,40,24 \mathrm{~mm}$ correspondent sensiblement aux dimensions définies par tamisage.

Dans chacune des catégories, les valeurs minimale et maximale des diamètres intermédiaires sont les suivantes:

\begin{tabular}{|c|c|c|c|}
\hline \multirow{2}{*}{ Catégomes } & \multirow{2}{*}{$\begin{array}{l}\text { DIAMĖTRE } \\
\text { INTER- } \\
\text { MÉDIAHRE } \\
\text { MOYEN } \\
\text { (mm) }\end{array}$} & \multicolumn{2}{|c|}{ D) AMẼTRE } \\
\hline & & minimal & maximal \\
\hline $\mathrm{I}$ & 60 & 50 & 75 \\
\hline II & 40 & 35 & 45 \\
\hline HII & 24 & 22 & 25 \\
\hline
\end{tabular}

Chacune des trois catégories est repérée par ses diametres intermédiaires extrèmes sur la courbe granulométrique moyenne des alluvions du Rhône (fig. 1).

Le poids spécifique moyen des galets est de 1,6 pesé sous l'eau.

\section{III. - Immersion des galets}

Les galets ont été immergés le 10 décembre 1959 en trois points repérés A, B, C sur la tigure 2.

- en A les galets catégorie I,

- en B les galets catégorie II,

- en C les galets catégorie III.

\section{IV. - Détection des galets}

La détection s'est effectuée à l'aide de cinq compteurs Geiger du type 13 G 14 montés dans une sonde étanche en forme de $V$ déplacée et maintenue à proximité du fond à partir d'un bateau (fig. 3). Les compleurs sont reliés à un intégrateur connecté à un microampèremètre enregistreur qui décèle le passage de la sonde à proximité d'un galet marqué; le repérage du bateau par triangulation sur des points de rive permet ainsi de siluer la position du galet.

rrois campagnes de détection ont été effectuces:

- La première du 16 au 18 décembre 1959,

- La seconde du 8 au 21 janvier 1960 ,

toutes deux intéressant les trois catégories de galets;

- La troisième s'est effectuée en deux phases :

- du 16 au 18 mars 1960 pour les galets de catégorie $I$,

- du 6 au 13 avril 1960 pour les galets de catégories II et III.

L'évolution du débit du Rhòne, pendant la période comprise entre l'immersion et la fin de la troisieme détection, est représentée sur la figure 4 .

\section{V. - Repérage des galets}

Les zones de dispersion des galets détectés au cours des deux dernières campagnes sont représentées sur la figure 2 .

\section{Première constatation :}

Les galets déplacés de leur point d'immersion sont repérés dans certains cas par unité; c'est ainsi que la troisième campagne de détection a permis de repérer au moins $80 \%$ des galets de la catégorie III.

Une partie des galets de catégorie II est entraince vers les épis situés en R.D. et dans ce cas leur détection est plus difficile.

\section{Deuxième CONSTATATron :}

Certains galets de la catégorie I sont restés à leur point d'immersion malgré les débits relativement forts survenus pendant la période expérimentale: $2750 \mathrm{~m}^{3} / \mathrm{s}$ le 4 janvier 1960 , $3200 \mathrm{~m}^{3} / \mathrm{s}$ le 6 mars 1960 ; la crue maximale en année moxenne est de l'ordre de $2700 \mathrm{~m}^{3} / \mathrm{s}$.

Des crues supérieures aux crues moyennes

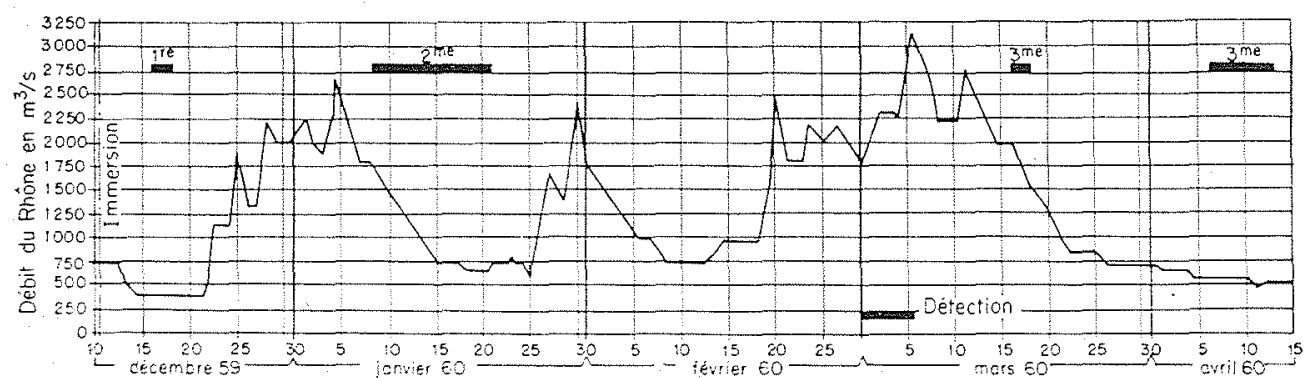

Fig. 4

Evolution du débit an cours de l'expérience "Traceurs». 
sont donc incapables de déplacer des galets de dimensions au maximum égales à $75 \mathrm{~mm}$ alors que le $a_{90}$ des galets atteint $90 \mathrm{~mm}$ environ; autrement dit, en année moyenne le charriage n'est pas généralisé; l'application de la formule de Meyer-Peter sous son aspect classique est de ce fait impossible pour le calcul du débit solide.

Pour tourner la difficulté, nous avons cependant tenté de faire le calcul avec cette mème formule en I'appliquant successivement à une suite de domaines partiels couvrant l'étendue du domaine granulométrique des galets; cette méthode, que nous justifierons à la fin de cet exposé, nécessite la connaissance de la force tractrice moyemne de début d'entrainement des galets constituant chacun des domaines granulométriques considérés.

Les résultats de l'expérience "traceurs» et d'une campagne de mesure au sondeur hydrophone permettent de déterminer la force tractrice de début d'entrainement des trois catégories de galets immergés.

\section{FORCES TRACTRICES DE DEBUT D'ENTRAINEMENT DES GALETS}

\section{I. - Interprétation des résultats des deuxième et troisième campagnes de détection}

- Après la crue de $2700 \mathrm{~m}^{3} / \mathrm{s}$, certains galets de la catégorie I sont restés au point $A$ d'immersion.

- Les galets de la catégorie II se sont tous très nettement déplacés.

- Les galets de la catégorie III se sont tous déplacés; certains sont cependant restés à proximité immédiate de leur point $\mathrm{C}$ d'immersion.

On pent a priori poser que les gros galets se déplacent moins aisément que les galets de plus faible dimension, ce qui permet de dire, d'après les remarques précédentes, que les conditions d'écoulement au maximum de la crue de $2700 \mathrm{~m}^{3} / \mathrm{s}$ étaient telles :

a) que les plus gros galets de la catégorie I n'ont pas été entraînés;

b) que les plus gros galets de la catégorie II ont tous été entrainés;

c) que les plus gros galets de la catégorie III étaient à la limite de leur stabilité.

Si $\tau_{1}$ est la force tractrice de début d'entrainement d'un galet de diamètre $D$ et - la force exercée sur ce galet, on peut écrire:

- Galets de catégorie $I$ :

$\left(D_{\max }=75 \mathrm{~mm}, \mathrm{D}_{\min }=50 \mathrm{~mm}\right)$ :

$\tau_{\tau 5}>\tau$
$\tau_{50}<\tau$ au point A pour $2700 \mathrm{~m}^{3} / \mathrm{s}$

- Galets de catégorie II:

$\left(\mathrm{D}_{\mathrm{inax}}=45 \mathrm{~mm}\right)$ :

$\tau_{4 ;}<\tau$ au point $B$ pour $2700 \mathrm{~m}^{3} / \mathrm{s}$;
- Galets de catégorie III :

$\left(\mathrm{D}_{\max }=25 \mathrm{~mm}\right)$ :

$\tau_{25} \# \tau$ au point $\mathrm{C}$ pour $2700 \mathrm{~m}^{3} / \mathrm{s}$.

De mème, les résultats de la troisième campagne de détection montrent qu'après la crue de $3200 \mathrm{~m}^{3} / \mathrm{s}$, tous les galets se sont déplacés, sauf les plus gros galets de la catégorie I. On peut alors écrire :

- Galets de catégorie I:

$\tau_{\tau 5}>\tau$ au point A pour $3200 \mathrm{~m}^{3} / \mathrm{s}$;

- Galets de catégorie $I I$ :

$\tau_{.5}<\tau$ au point B pour $3200 \mathrm{~m}^{3} / \mathrm{s}$;

- Galets de catégorie III :

$\tau_{25}<\tau$ au point $\mathrm{C}$ pour $3200 \mathrm{~m}^{3} / \mathrm{s}$.

Notons, pour compléter les renseignements que la campagne de mesure avec traceurs nous a apportés, que des sondages efrectués en mars 1957 à l'aide d'un détecteur hydrophonique dans la région de Pierre-Bénite, ont montré que le tout début d'entrainement des galets avait lieu pour un débit voisin de $1500 \mathrm{~m}^{3} / \mathrm{s}$.

\section{II. - Détermination des forces tractrices aux points d'immersion \\ pour les débits de 2700 et $3200 \mathrm{~m}^{3} / \mathrm{s}$}

Les pentes moyennes de la ligne d'énergie pour les débits de $2700 \mathrm{~m}^{3} / \mathrm{s}$ et $3200 \mathrm{~m}^{3} / \mathrm{s}$ dans la région du lit intéressée par l'expérience sont les suivantes :

$$
\begin{array}{ll}
\mathrm{Q}=2700 \mathrm{~m}^{3} / \mathrm{s} & J=0,325 \cdot 10^{-3} \\
\mathrm{Q}=3200 \mathrm{~m}^{3} / \mathrm{s} & J=0,342 \cdot 10^{-3}
\end{array}
$$

Si l'on tient compte du fait que la perte d'énergie totale de l'écoulement est due au frottement sur les berges, aux irrégularités des 
fonds (dunes, épis) et, ce qui nous intéresse, au travail dépensé pour le déplacement des matériaux de fond, l'expérience montre que la pente $J_{e}$ de la ligne d'énergie à prendre en compte est égale à :

$$
J \frac{\mathrm{Q}_{s}}{\mathrm{Q}}\left(\frac{k_{s}}{k_{r}}\right)^{3 / 2} .
$$

$Q$ est le débit total;

$Q_{s}$ est le débit qui participe au charriage sur le fond;

$\mathrm{k}_{s}$ est le coefficient global de rugosité de Strickler;

$k$, est le coefficient de rugosité de Strickler des matériaux de fond.

$k$. est de l'ordre de 40 , c'est la valeur obtenue par la relation connue :

$$
k=\frac{26}{\mathrm{D}_{90}{ }^{1 / 6}}
$$

e'est aussi la valeur déterminée à partir des caractéristiques des écoulements à faible débit dont l'énergie n'est effectivement dépensée que par frottement sur les galets.

$k_{s}$ est aisément calculable pour les débits considérés :

$$
k_{s}=\frac{\mathrm{Q}}{\mathrm{SR}^{2 / 3} J^{1 / 2}}
$$

$\mathrm{Q}_{s} / \mathrm{Q}$ est plus délicat à déterminer; nous sommes restés dans la gamme des valeurs calculables dans des cas simples, et pour 2700 ou $3200 \mathrm{~m}^{3} / \mathrm{s}$ nous avons adopté $\left(\mathrm{Q}_{s} / \mathrm{Q}\right)=0,86$; l'erreur relative probable sur cette valeur n'excède certainement pas $10 \%$.

En définitive, les pentes $J_{G}$ calculées pour les débits de 2700 et $3200 \mathrm{~m}^{3} / \mathrm{s}$ sont sensiblement les mêmes et égales à $0,24 \cdot 10^{-3}$ à moins de $10 \%$ près.

Les forces tractrices $\tau_{2}$ aux points $A, B, C$ d'immersion, égales à $h \mathrm{~J}_{e}$ ( $h$ hauteurs d'eau en mètres), sont les suivantes:

\begin{tabular}{|c|c|c|c|}
\hline $\mathrm{Q}$ & \multicolumn{3}{|c|}{$\tau$ (tonnes $\left./ \mathrm{m}^{2}\right)$} \\
\cline { 2 - 3 } $\mathrm{m}^{3} / \mathrm{s}$ & $\mathrm{A}$ & $\mathrm{B}$ & $\mathrm{C}$ \\
\hline 2700 & $1,78.10-3$ & $1,76.10-3$ & $1,40.10^{-3}$ \\
3200 & $1,93.10-3$ & $1,91.10-3$ & $\mathbf{1 . 5 4 . 1 0 ^ { - 3 }}$ \\
\hline
\end{tabular}

En outre la force tractrice maximale pour le débit de $1500 \mathrm{~m}^{3} / \mathrm{s}$ à partir duquel on détecte un début de charriage est :

$\tau=h_{\mathrm{max}} \mathrm{J}_{e}=6,15 \cdot 0,238 \cdot 10^{-3}=1,50 \cdot 10^{-3} \mathrm{t} / \mathrm{m}^{2}$
En admettant comme peu probable que ce début de charriage concerne des éléments supérieurs à $45 \mathrm{~mm}$, les inégalités citées précédemment s'écrivent alors de la façon suivante:

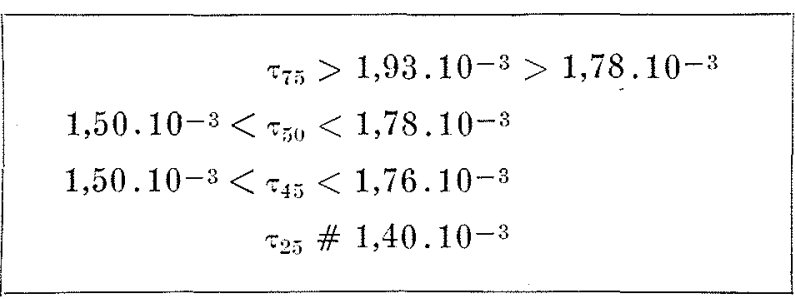

On peut encore écrire, en supposant les valeurs de - peu différentes des moyennes arithmétiques des deux membres de chaque inégalité :

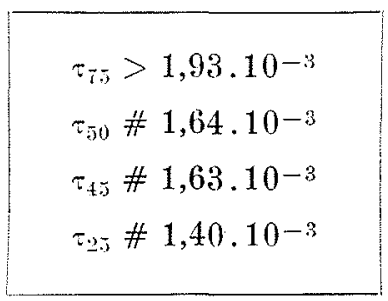

A partir de ces valeurs moyennes, le coefficient d'entraînement $A$, qui entre dans la relation admise par de nombreux auteurs:

$$
\tau=\mathrm{A} \bar{\omega}^{\prime \prime} \mathrm{D},
$$

a été calculé pour les différents diamètres :

- Pour D $=75 \mathrm{~mm}$ :

$$
\begin{aligned}
A>\frac{1,93 \cdot 10^{-3}}{\bar{\omega}_{s}^{\prime \prime} \times 75 \cdot 10^{-3}} & \\
= & 0,016 \text { avec } \bar{\omega}^{\prime \prime}:=1,6 \mathrm{t} / \mathrm{m}^{3} ;
\end{aligned}
$$

- Pour $\mathrm{D}=50 \mathrm{~mm}$ :

$$
\mathrm{A} \#=\frac{1,64 \cdot 10^{-3}}{\bar{\omega}^{\prime \prime} \times 50 \cdot 10^{-3}}=0,0205 ;
$$

- Pour $\mathrm{D}=45 \mathrm{~mm}$ :

$$
\text { A \# 0,0226; }
$$

- Pour D $=25 \mathrm{~mm}$ :

$$
\text { A \#0,0350. }
$$

\section{III. - Premières conclusions tirées des essais}

1. En admettant que pour $\mathrm{D}=75 \mathrm{~mm}$, A ne dépasse pas la valeur 0,02 , on constate que $A$ est décroissant au fur et à mesure que le diamètre augmente pour tendre probablement vers une valeur constante peu différente de 0,02 . 
Autrement dit, la stabilité relative des galets varie en raison inverse de leur diamètre, ce qui peut s'expliquer par la «protection» qu'assurent les gros galets vis-à-vis des galets de moindre dimension.

2. Pour les débits inférieurs à $1500 \mathrm{~m}^{3} / \mathrm{s}$, $1300 \mathrm{~m}^{3} / \mathrm{s}$ par exemple, aucun charriage n'est détecté; à ce débit de $1300 \mathrm{~m}^{3} / \mathrm{s}$, la force tractrice maximale dans la région intéressée par l'expérience est de $1,30 \cdot 10^{-3} \mathrm{t} / \mathrm{m}^{2}$ environ; si l'on adopte une valeur constante du coefficient d'entrainement, soit $\mathrm{A}=0,0350$ par exemple, tous les galets dont le diamètre est inférieur à :

$$
\mathrm{D}=\frac{1,30.10^{-3}}{\bar{\sigma}^{\prime \prime}{ }_{s} \times 0,035}=24 \mathrm{~mm}
$$

devraient se déplacer; or il n'en est rien, ce qui confirme bien la première remarque : A ne peut être constant; pour les faibles diametres, il croît au fur et à mesure que $D$ décroît.

Nous verrons quelle loi $\mathrm{A}=f(\mathrm{D})$ il est possible d'adopter, puis, de quelle facon utiliser les résultats trouvés pour déterminer le débit solide de charriage à partir des hypothèses et des résultats expérimentaux exposés au chapitre suivant.

\section{HYPOTHĖSES POUR LE CALCUL DU DÉBIT SOLIDE DANS LE DOMAINE DU CHARRIAGE PARTIEL}

Soit $\tau_{x}$ la répartition de force tractrice dans une section transversale moyenne du lit de la rivière.

$\tau_{0}$ la force tractrice de début de charriage d'une catégorie de galets $\mathrm{D}_{0} \mathrm{D}_{1}$.

$n$ le pourcentage en poids des galets de catégorie $\mathrm{D}_{0} \mathrm{D}_{1}$, c'est-à-dire compris entre les diamètres $\mathrm{D}_{0}$ et $\mathrm{D}_{1}$. $n$ est déterminé à partir de la courbe granulométrique moyenne de l'ensenble des alluvions.

Le débit solide tolal $g$, dans l'hypothèse ò le lit serait constitué uniquenent de galets de catégorie $\mathrm{D}_{0} \mathrm{D}_{1}$, est de la forme :

$$
g=\int_{x=0}^{a=l} \sum_{i}\left(\tau-\tau_{0}\right)^{3 / 2} d x
$$

Fig. 5

Granulométrie des alluvions sur le modèle de la Tinée.

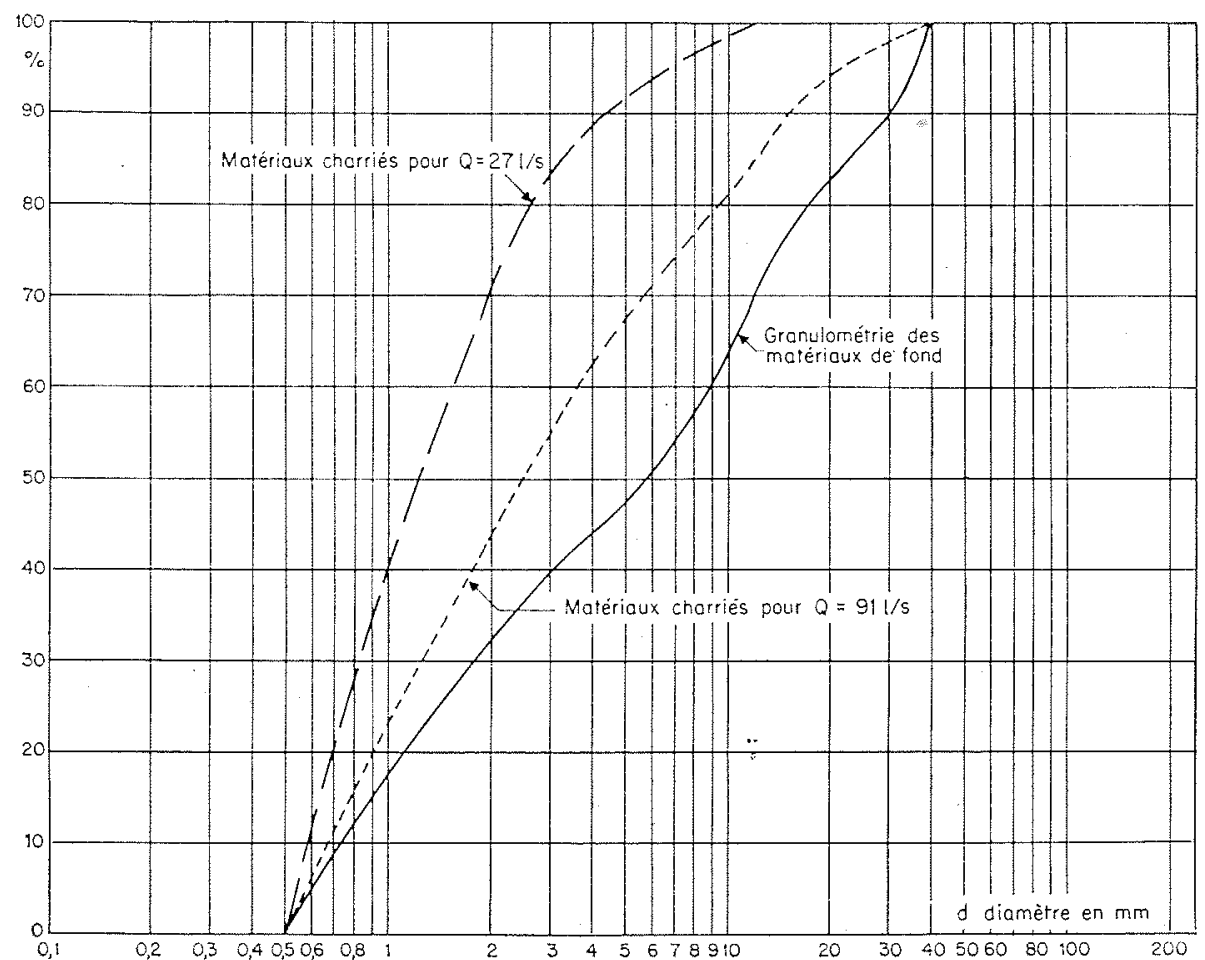


en désignant par $l$ la largeur du lit sur laquelle $\tau$ est plus grand que $\tau_{0}$. En fait, le pourcentage en poids de la catégorie $\mathrm{D}_{0} \mathrm{D}_{1}$ étant $n$, il paraît logicne d'adopter :

$$
g_{\mathrm{D}_{0} \mathrm{D}_{2}}=n \int_{x=0}^{x=2} k\left(\tau-\tau_{0}\right)^{3 / 2} d x
$$

Ce mème calcul peut être répété pour chacune des catégories $\mathrm{D}_{0} \mathrm{D}_{1}, \mathrm{D}_{1} \mathrm{D}_{2}$ etc., couvrant l'ensemble du domaine granulométrique; le débit solide total est alors égal à la somme des débits partiels ainsi calculćs :

$$
\mathrm{G}=\Sigma g
$$

Application de LA Méthode a UN CAS CONCRET.

Une telle méthode est-elle justifiée?

Pour la vérifier, nous l'avons appliquée au calcul du débit solide d'un torrent dont la représentation sur modèle à une échelle relativement grande, $1 / 12,5$, avait permis l'étude expérimentale précise des débits solides précisément dans le domaine du charriage partiel. Il s'agit de la Tinée à Isola représentée sur modèle pour l'étude de la chute de Valabres.

Pour le détail de cette étude nous reportons à la note de MM. Braudeau et Nizery parue dans les «Proceedings» de septembre 1953 et intitulée «Variation in grain size distribution of bed load in a river section $\gg$.

La courbe granulométrique des alluvions de la Tinée est représentée sur la figure 5.

Pour un débit de $27 \mathrm{l} / \mathrm{s}$ modèle, l'expérience a montré que le débit solide total du modèle était de $0,019 \mathrm{~kg} / \mathrm{s}$; les débits solides partiels

\begin{tabular}{|c|c|c|}
\hline$\underset{(\mathrm{mm})}{\mathrm{D}}$ & $\begin{array}{l}\% \text { EN POIDS } \\
\text { DES ELLEMENTS } \\
\text { CHARRÉS }\end{array}$ & $\begin{array}{l}\text { DÉmir soLIDE } \\
\quad(\mathrm{kg} / \mathrm{s})\end{array}$ \\
\hline $\begin{aligned} 30 & <\mathrm{D}<40 \\
20 & <\mathrm{D}<30 \\
10 & <\mathrm{D}<20 \\
5 & <\mathrm{D}<10 \\
3 & <\mathrm{D}<5 \\
2 & <\mathrm{D}<3 \\
1 & <\mathrm{D}<2 \\
0,5 & <\mathrm{D}<1\end{aligned}$ & $\begin{array}{r}0 \\
0 \\
\varepsilon \\
7 \\
10 \\
13 \\
30 \\
40\end{array}$ & $\begin{array}{ll}0 & \\
0 & \\
\varepsilon & \\
1,3 & 10^{-3} \\
1,9.10^{-3} \\
2,5 & 10^{-3} \\
5,7.10^{-3} \\
7,6.10^{-3}\end{array}$ \\
\hline Total & $100 \%$ & $19.10^{-3}$ \\
\hline
\end{tabular}
par tranche granulométrique sont les suivants:

Sans entrer dans le détail des calculs, disons qu'en appliquant la méthode proposée, nous avons cherché à déterminer la valeur de $\tau_{0}$ pour chacune des catégories de galets : $30<D<40$, $20<\mathrm{D}<30$, etc., de façon à ce que les valeurs du débit solide calculé par catégorie de galets soient égales aux valeurs expérimentales trouvées.

Les valeurs de $\tau_{0}$ ainsi déterminées et les valeurs du coefficient d'entrainement qui s'en

\begin{tabular}{|c|c|c|c|}
\hline $\mathrm{D}_{m}$ & $\begin{array}{c}\tau_{0} \\
\left(\mathrm{t} / \mathrm{m}^{2}\right)\end{array}$ & $\begin{array}{l}\mathrm{D}_{\text {mogen }} \\
(\mathrm{mm})\end{array}$ & $\begin{array}{l}A= \\
\frac{\tau}{(t)^{\prime \prime} D_{\text {moyen }}}\end{array}$ \\
\hline $\begin{array}{l}30<\mathrm{D}<40 \\
20<\mathrm{D}<30\end{array}$ & $>4,8 \cdot 10^{-4}$ & $\begin{array}{l}35 \\
25\end{array}$ & $>0,012$ \\
\hline $10<\mathrm{D}<20$ & $4,8 \cdot 10^{-1}$ & 15 & 0,020 \\
\hline $5<\mathrm{D}<10$ & $2,7 \quad .10-4$ & 7,5 & 0,0225 \\
\hline $3<\mathrm{D}<5$ & $2,1 \cdot 10^{-4}$ & 4 & 0,0327 \\
\hline $2<D<3$ & $1,85.10^{-4}$ & 2,5 & 0,0460 \\
\hline $1<\mathrm{D}<2$ & $1,85 \cdot 10^{-1}$ & 1,5 & 0,077 \\
\hline $0,5<\mathrm{D}<1$ & $1,75 \cdot 10^{-4}$ & 0,75 & 0,145 \\
\hline
\end{tabular}
déduisent sont les suivantes :

Les valeurs de $\tau_{0}$ trouvées sont acceptables si le calcul du débit solide effectué avec ces mêmes valeurs et pour des conditions d'écoulement différentes donne des résultats proches des résultats expérimentaux.

Pour un débit de $91 \mathrm{l} / \mathrm{s}$, en admettant une valeur constante $0,02 \mathrm{du}$ coefficient A pour les catégories $20<\mathrm{D}<30$ et $30<\mathrm{D}<40$, les calculs ont donné les résultats suivants, que nous

\begin{tabular}{|c|c|c|}
\hline $\begin{array}{c}\mathrm{D} \\
(\mathrm{mm})\end{array}$ & $\begin{array}{c}\text { DÉBIT SOLIDE } \\
\text { MESURS EN } \\
\left(10^{-2} \mathrm{~kg} / \mathrm{s}\right)\end{array}$ & $\begin{array}{c}\text { DÉBIT SOLIDE } \\
\text { CALCULÉ } \\
\left(10^{-2} \mathrm{~kg} / \mathrm{s}\right)\end{array}$ \\
\hline $\begin{aligned} 30 & <\mathrm{D}<40 \\
20 & <\mathrm{D}<30 \\
10 & <\mathrm{D}<20 \\
5 & <\mathrm{D}<10 \\
3 & <\mathrm{D}<5 \\
2 & <\mathrm{D}<3 \\
1 & <\mathrm{D}<2 \\
0,5 & <\mathrm{D}<1\end{aligned}$ & $\begin{array}{l}0 \\
2,25 \\
6,75 \\
5,85 \\
5,40 \\
4,95 \\
9,45 \\
10,35\end{array}$ & $\begin{array}{r}0 \\
0,4 \\
5,2 \\
10,4 \\
5,8 \\
5,3 \\
12,9 \\
13\end{array}$ \\
\hline Total & $45-10-2 \mathrm{~kg} / \mathrm{s}$ & $53.10^{-2-2} \mathrm{~kg} / \mathrm{s}$ \\
\hline
\end{tabular}
comparons aux valeurs expérimentales :

On constate localement certains écarts, mais les débits solides globaux expérimentaux ef calculés sont équivalents à $15 \%$ près. Cette différence relativement faible entre l'expérience et le calcul nous a paru justifier l'utilisation de notre méthode au calcul du charriage du Rhône.

Une première synthèse des études et expériences qui viennent d'ètre relatées est auparavant nécessaire :

1. Il apparait que les premières conclusions tirées des expériences «traceurs» dans le Rhône se confirment; en effet $A$ croît inversement au 
diamètre et tend vers la même limite 0,02 pour les plus gros éléments.

2. Les éléments les plus fins de la courbe granulométrique commencent à se déplacer pour une même valeur de la force tractrice :

$$
1,80 \cdot 10 \div \mathrm{t} / \mathrm{m}^{2}
$$

environ dans le cas particulier du modèle de la Tinée à Isola. C'est-ă-dire que pour les valeurs du dianètre $d$ inférieures à une certaine limite $D$, le coefficient d'entrainement $A$ est une fonction hyperbolique de $d$ :

$$
\mathrm{A}=\frac{\tau_{0}}{\bar{\omega}^{\prime \prime} d} \text {. }
$$

Cette observation est importante; en effet la force tractrice de tout début de charriage du Rhône est, nous l'avons noté précédemment, de l'ordre de 1,40 a $1,50.10^{-3} \mathrm{t} / \mathrm{m}^{2}$; la force tractrice de début d'entraînement des galets de
$25 \mathrm{~mm}$ est précisément de $1,40.10^{-3} \mathrm{t} / \mathrm{m}^{2}$, c'està-dire que tous les galets de diamètre inférieur a $25 \mathrm{~mm}$ se déplacent simultanément pour une valeur de $\tau_{0}$ égale à $1,40 \cdot 10^{-3} \mathrm{t} / \mathrm{m}^{2}$.

Ces constatations permettent de compléter les données nécessaires au calcul du charriage du Rhône à Pierre-Bénite, en particulier d'établir la relation $\tau_{0}=j(D)$.

$$
\begin{aligned}
& \text { pour } \left.\mathrm{D} \geqslant 50 \mathrm{~mm} \tau_{0}=0,02 \bar{\omega}^{\prime \prime} \mathrm{D}(\mathrm{D}) \text { en mètres }\right) \\
& =0,032 \mathrm{D} \\
& \text { pour } \mathrm{D}=45 \mathrm{~mm} \tau_{0}=1,63.10^{-3} \mathrm{t} / \mathrm{m}^{2} \\
& \text { pour } \mathrm{D} \leqslant 25 \mathrm{~mm} \tau_{0}=1,40 \cdot 10^{-3} \mathrm{t} / \mathrm{m}^{2}
\end{aligned}
$$

VALEURS DU DÉBIT SOLIDE DU RHONE.

En définitive, les valeurs calculées du débit solide du Rhône à Pierre-Bénite pour cing catégories de galets et en fonction du débit liquide, sont les suivantes :

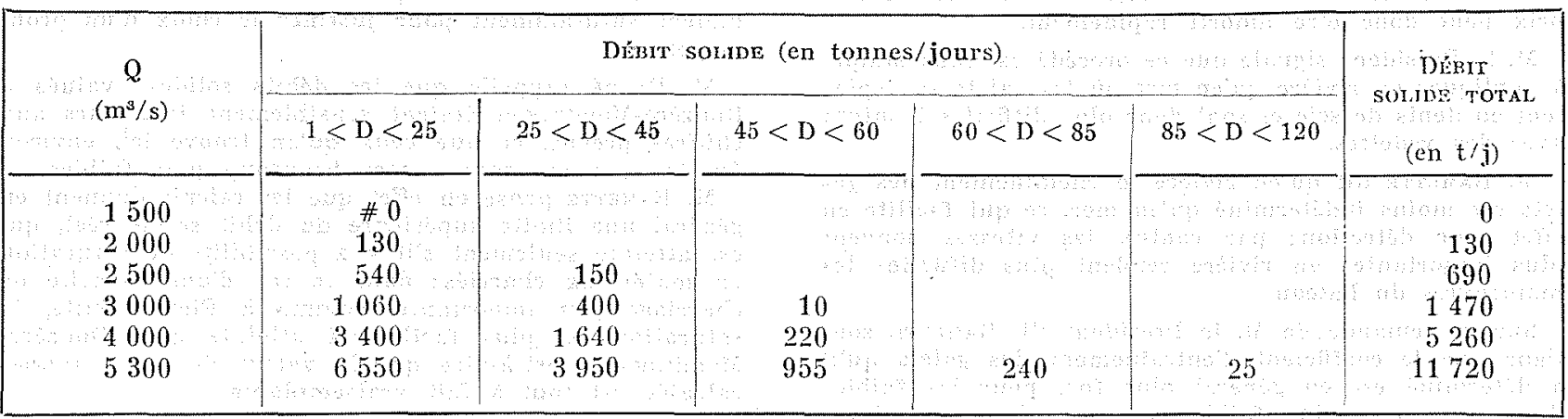

Le débit solide annuel, calculé à partir de la courbe moyenne des débits liquides classés, serait ainsi de 35000 tonnes; ce chiffre est très faible comparé à la valeur du débit solide dans le tiers central du Rhône, qui a été évalué par voies expérimentale et théorique à 700000 tomnes en moyenne, les pentes plus faibles et les débits moins élevés du Rhône dans la région lyonnaise expliquent cette diférence de capacité de charriage.

\section{CONCLUSION}

L'expérience réalisée dans le Rhône à l'aide de galets marqués a done permis de metre en évidence qu’à l'intérieur d'une famille de galets ayant une granulométrie suffisamment étendue, le coefficient d'entraînement $A$ varie en raison inverse du diamètre et tend vers une limite voisine de 0,020 pour les galets les plus gros. Une telle conclusion n'est d'ailleurs pas en contradiction avec ce qui est connu, dire par exemple, avec Meyer-Peter, que le début de charriage total a lieu pour une force tractrice :

$$
\tau_{0}=0,047 \omega^{\prime \prime} l_{\text {nicien }}
$$

suppose que les galets dont le diametre correspond au $d_{90}$ par exemple se déplacent; $d_{90}$ étant plus grand que $d_{\text {muen }},{ }^{\wedge} \rightarrow$ donc bien inférieur à $0,04.7$.

A ce propos, il nous paraitrait plus juste de définir la valeur de $\tau_{0}$ non pas a partir de $d_{\text {rivgen }}$ qui est fonction de la courbe granulométrique toujours très indéterminée dans la gamme des faibles diamètres, mais à partir d'une dimension caractéristique des gros diamètres, en général mieux connus, et par exemple du $d_{95}$.

On aurait ainsi $\tau_{0}=0,020 \bar{\omega}^{\prime \prime} d_{95}$, expression 
plus satisfaisante, qui exprime que, pour cette valeur de $\tau_{0}$, les galets de diamètre égal à $d_{95}$ sont à la limite de leur stabilité et que $\tau_{0}$ correspond bien en conséquence au seuil du charriage généralisé.

Par ailleurs, l'application de la méthode de calcul du débit solide au cas particulier de la Tinée est satisfaisante précisément si le coefficient d'entrainement varie en fonction du dia- mètre des galets selon une loi analogue à celle qui a été déduite de l'expérience «traceurs ». On ne peut prétendre a priori qu'une telle analogie justifie dans tous les cas les hypothèses de calcul formulées; elle est cependant suffisamment nette dans le cas étudié pour que se poursuive la vérification de la méthode de calcul proposée, qui pourra sans doute être complétée ou améliorée.

\section{I S C U S I O N}

Président: M. de RouviLLe

M. Ie Président remercie M. LAMETre qui a présenté la communication et estime que la tcchnique exposée requiert des moyens financiers assez importants.

M. Rametre indique que cette technique nécessite effectivement un matériel assez coûteux; ce matẻriel, maintenant mis au point, est cependant utilisable pour de nombreuses détections en mer ou en rivière; son prix peut donc être amorti rapidement.

M. le Président signale que ce procédé est plus simple à appliquer en rivière qu'en mer où les galets se déplacent en dents de scie et sont done plus difficiles à suivre avec des vedettes.

M. Rametre dit qu'en rivière le cheminement des galets est moins indéterminé qu'en mer, ce qui facilite en effet leur détection; par contre, les vitesses souvent plus importantes en rivière rendent plus difficiles les manœuvres du bateau.

Sur la demande de M. le Président, M. Rametre souligne que le coefficient d'entrainement des galets qu'il a déterminé est en général plus fort pour les faibles diamètres mais plus faible vers les diamètres élevés que la valeur donnée par Meyer-Peter : 0,047, qui entre dans la relation $\tau_{0}=0,047 \quad \omega^{\prime \prime}{ }_{s} d_{\text {mosen }}$; c'est-à-dire qu'en moyenne il ne semble pas y avoir de contradiction. La formule de charriage de Meyer-Peter reste valable lorsque les forces tractrices sont sensiblement supérieures à celles de début d'entrininement de tous l'es galets; lorsqu'elles en sont voisines ou inférieures, il est nécessaire de considérer les conditions d'entraînement des différentes Lranches granulométriques; c'est ainsi que l'on a constaté que les gros yalets étaient relativement moins stables que les petits galets.

M. le Président pense qu'il y a une grosse influence de l'imbrication des galets en surface

M. Ramerte répond qu'il y a, en effet, lorsque le charriage n'est pas généralisé, un effet de « pavage» des gros éléments; ce même phénomène est d'aillenrs observé sur morlèle; les hypothèses formulées pour le calcul du débit solide en tiennent impicitement compte, puisque les comparaisons entre le calcul et l'expérience sont satisfaisantes; il est certain qute de nombreuses applications de la methode proposée seront encore né. cessaires avant de pouvoir tirer une conclusion définitive s'il en est une dans ce domaine complexe.

M. PardÉ estime que le charriage annuel de 35000 tonnes, trouvé par M. Rametre à Pierre-Bénite, est très peu élevé.

M. Ramette dit que le faible charriage du Rhône dans la rẻgion de Pierre-Bénite est confirmé par l'observation: une souille de dragage creusée dans le Rhòne en umont du confluent avec la Sâne ne recueille que fort peu d'alluvions: la Saône n'apporte, elle aussi, que peu d'éléments; la majorité des éléments charriés en aval du confuent est arrachée aux fonds qui se creusent progressivement.

M. Rametre, sur la demande de M. Pardé, dit que le calcul du débit solide a été effectué sur un tronçon de $1 \mathrm{~km}$ environ, dont les profils en travers se ressemblaient suffisamment pour justifier le choix d'un profil moyen.

M. Pandé rappelle que les débits solides évalués á Donzère-Mondragon étaient sensiblement inférieurs aux chiffres prévus, et que ceux qu'on trouve ici, environ $30000 \mathrm{t}$ par an, sont encore beaucoup plus faibles.

M. Ramette pense en effet que les calculs donnent en général une limite supérieure du débit solide réel, qui est atteinte seulement s'il y a possibilité de saturation en matériaux charriés; dans le cas d'une capacité de charriage pen importante, comme à Pierre-Bénite, la saturation est plus facilement atteinte qu'à DonzèreMondragon, c'est-à-dire que la valeur de 35000 tomnes calculée est tout à fait vraisemblable.

M. Grataning rappelle d'abord les raisons pour lesquelles la Compagnie Nationale du Rhône a entrepris, au voisinase de la future chute de Pierre-Bénite, une campagne spéciale de mesures basées sur le cheminement des galets radioactifs.

La seule mesure directe du débit solide de charriage qui ait été entreprise jusqu'ici sur le fleuve, a été rendue possible par la présence des barrages de retenue du tiers central du Bas-Rhône. Nous avons déjà rendu compte au Comité Technique de la S.H.F. (sénnce du 28 juin 1957 ) du résultat des cinq premières années de mesures faites à Donzère. Le charriage moyen annuel ainsi déterminé par le sondage périodique des fonds a été de l'ordre de $350000 \mathrm{~m}^{3}$ pendant la période 1952-1957. De. puis, les observations continuent tant à Donxère qu'à Montélimar et à Baix-le-Logis-Neuf, et on a entrepris de plus une étude systématique du débit solide de suspension en amont et en aval du confluent de l'Isère.

Grâce aux mesures faites, on a pu déterminer les constantes d'ajustement qui permettent l'application de la formule de Meyer-Peter dans la région de Donzère (voir communication D 21 au $7^{\prime}$ Congrès de I'A.I.R.H.) Applique brutalement à Pierre-Bénite, cette formule n'aurait donné que des résultats décevants pour la raison suivante. Le débit solide est en effet fonction de la différence de deux termes. L'un fait intervenir la force tractrice $\tau$ pour le débit considéré; l'autre, la force tractrice $\tau$ co correspondant au débit liquide $Q_{0}$ de début d'entraînement. Or, les crues qui complent le plus, dans le charriage annuel moyen, ne sont pas, comme on serait tenté de le croire, les plus grandes crues, mais les crues moyennes, de beaucoup les plus fréquentes, et qu'on 
peut appeler pour simplifier «crues efficaces». Comple tenu de la fréquence respective des différents débits aux deux stations considérées, le débit efficace $Q_{c}$ est de l'ordre de $2000 \mathrm{~m}^{3} / \mathrm{s}$ à Pierre-Bénite situé en amont, et de $3000 \mathrm{~m}^{3 / \mathrm{s}}$ à Donzère, où le bassin versant est plus important. Ces débits sont entre eux approximativement dans le même rapport que les débits moyens. O1' des essais à l'hydrophone ont montré qu'il n'en est pas de même pour les débits de début d'entrainement $Q_{0}$; ceux-ci sont pratiquement égaux $\left(1500 \mathrm{~m}^{3} / \mathrm{s}\right.$ ) pour les deux stations, les pentes étant beaucoup plus faibles à Pierre-Bénite qu'à Donzère.

Il en résulte les conséquences suivantes : à Donzère, où l'écart $Q_{0}-Q_{0}$ est notable $\left(1500 \mathrm{~m}^{3} / \mathrm{s}\right)$, le charriage moyen, qui est une fonetion rapidement croissante de $\tau_{0}-\tau$, est important, et la formule de Meyer-Peter le donne avec une bonnc précision. A Fierre-Bénite, où Q. - $-Q_{0}$ est plus modeste $\left(500 \mathrm{~m}^{3} / \mathrm{s}\right)$, le charriage est faible sans doute (il semble qu'il soit de l'ordre du vingtième du précédent), mais la formule le donne avec très faible précision. C'est pourquoi il y avait lieu de procéder ì une détermination directe du débit solide a Pierre-Bénite même, et notamment une mesure précise de to.

Les mesures qui ont été faites, et dont $M$. Rametre a rendu compte, ont montré qu'il subsiste une deuxième source d'erreurs dans la détermination du charriage pour les débits peu supérieurs à $\mathrm{O}_{\mathrm{o}}$.

Il semble, en effet, que le coefficient d'entrainement $A$ ne soit pas constant comme on l'admet ordinairement, mais dépende du diamètre des grains. D'après les chiffres qui viennent d'être cités, quand le diamètre des matériaux double, la force tractrice de début d'entraînement $\tau$, au lieu d'augmenter de $100 \%$ comme on l'admet souvent, semble ne crôtte que de $30 \%$ sur la Tinée et de $15 \%$ à Pierre-Bénite. Il convient de remarcuer à ce sujet que, dans la granulométrie naturelle, il n'est pas exclu que la forme des grains varie en moyenne en fonction de la grosseur, ce qui pourrait expliquer certaines des diffèrences constatées.

Quoi qu'il en soit, lorsque le charriage à étudier correspond à une granulométrie étendue, et qu'on se trouve dans un domaine de débits voisins de $O_{0}$, il $\mathrm{y}$ a intérêt à ne pas se contenter d'appliquer une formule globale où intervient un seul diamètre utile (le diamètre moyen par exemple), comme on le fait habituellement, mais a étudier le cheminement de chaque classe de matériaux. C'est la méthode fine qui vient d'être préconisée ici.

Les mesures faites à Pierre-Bénite ont permis de préciser le problème qui avait été posé (détermination du débit solide de charriage pour les débits modérés du Rhône), mais elles ouvrent des perspectives nouvelles qui mériteraient d'être contirmées ou précisées par de nouvelles expériences dans la nature ou sur modèle réduit.

M. Canleux demande le pourcentage des galets retrouvés.

M. RaMETE: répond qu'on a retrouvé, environ, $80 \%$ de galets moyens. Dans la région des épjs, il était très difficile d'utiliser la sonde sans risque de l'endommager et l'on a détecté relativement moins de galets.

M. Ie président remercie et lélicite M. Rametre de cette importante communication.

recherche :

\section{JEUNE INGÉNIEUR HYDRAULICIEN}

ayant expérience hydraulique agricole

pour poste Paris, comportant missions outre-mer

Ecrire à B 306 PRESS, 31, boulevard Bonne-Nouvelle, Paris (2c) 